\title{
Percepción sobre el Derecho a la Salud y Acceso a Servicios en Usuarios del Régimen Subsidiado en un Municipio Colombiano, 2005
}

\author{
Subsidised Regimen users' perceptions concerning their right to \\ health and access to health services in a Colombian town, 2005
}

\author{
Nelson Alvis-Guzmán ${ }^{1}$, Luís Alvis-Estrada² y Julio Orozco-Africano ${ }^{1}$ \\ 1 Departamento de Investigaciones Económicas y Sociales-DIES. Universidad de Cartagena, \\ Colombia. E-mail: nalvis@yahoo.com \\ 2 Facultad de Medicina. Universidad de Cartagena, Colombia.
}

Recibido 5 Septiembre 2007/Enviado para Modificación 25 Abril 2008/Aceptado 1 Junio 2008

\section{RESUMEN}

Objetivo Determinar la percepción que los afiliados al Régimen Subsidiado de Salud tienen respecto al derecho a la salud y el acceso a los servicios.

Métodos Se llevó a cabo un estudio transversal en el que se aplicó un formulario previamente validado a 600 usuarios mediante muestreo aleatorio, ponderando la participación porcentual de los afiliados a las Empresas Promotoras de Salud-EPS del Régimen Subsidiado del municipio de Arjona, Bolívar. Los datos fueron tabulados y procesados utilizando el paquete estadístico SPSS $® 10.0$.

Resultados El desconocimiento y la falta de información de los procesos de gestión es evidente entre los usuarios del Régimen Subsidiado de Salud. Además, la percepción que tienen los usuarios acerca de la calidad de la atención de las EPS del RS se encuentra correlacionada positivamente con el nivel de participación de los afiliados en las actividades de dichas empresas.

Conclusiones Se carece de indicadores que midan el estado actual del derecho a la salud. En su defecto se usan cifras relacionadas con los recursos destinados a salud y su gestión. Así, los logros en materia de cobertura del aseguramiento en salud deben significar, no solo población asegurada, sino acceso efectivo a servicios de salud suficientes y de calidad. En tal sentido, la opinión de la comunidad puede constituirse en indicador del cumplimiento del derecho a la salud.

Palabras Clave: Derecho a la salud, participación comunitaria (fuente: DeCS, BIREME).

\section{ABSTRACT}

Objective Determining Subsidised Regime (EPS-RS) clients' perceptions regarding their right to health and access to health services.

Methods A cross-sectional study of 600 Subsidised Regime clients from the 
municipality of Arjona, Bolivar department, was carried out. A previously validated questionnaire was given to the randomly selected study population, weighting EPSRS affiliated clients' participation. The data was processing using the SPSS ${ }^{2} 10.0$ statistical package.

Results Ignorance of and lack of information regarding management processes were evident amongst EPS-RS clients. Furthermore, their perception about the quality of EPS-RS attention was positively correlated with affiliated clients' level of participation in these companies' activities.

Conclusions No indicators measuring the right to health are currently available. Figures related to the resources destined for health and managing it are thus used. Achievements in ensuring health insurance coverage should not just therefore refer to the nonassured population but to effective access to health services which are sufficient and of good quality. Community' opinion might thus become an indicator regarding how people's right to health is being complied with.

Key Words: Health, right, community participation (source: MeSH, NLM).

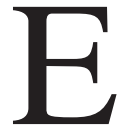

l derecho a la salud hace parte de los llamados derechos de "segunda generación” aunque también se conocen como Derechos Económicos, Sociales y Culturales-DESC- debido a que su ejercicio y cumplimiento solo se dará gracias a la interacción de las personas que hacen parte de la sociedad (1). Existen propuestas de clasificar estos derechos como morales, es decir, derechos con los cuales cuenta cualquier ser humano sin necesidad de que cumpla condiciones especiales o haya una ley que los respalde (2). En la actualidad estos derechos luchan por su legitimidad, sobretodo en un mundo donde prima el pensamiento neoliberal, y muchas veces son objeto de disputas judiciales.

Por otra parte, el derecho a la salud no sólo es entendido como derecho a estar sano, sino como libertades relacionadas con el manejo del cuerpo y derechos relacionados con sistemas de protección que otorgue oportunidades iguales para alcanzar el nivel de salud que permita vivir dignamente. Así, son elementos esenciales para su cumplimiento: la disponibilidad y accesibilidad a los servicios de salud (debe ser no discriminatoria, accesible económica y físicamente, y con amplia información) y la aceptabilidad y calidad en la prestación de tales servicios (3).

De igual modo, uno de los fines esenciales del Estado en Colombia, plasmado en la Constitución de 1991, es el de facilitar la participación de todos en las decisiones que suelen afectar la vida económica, política, administrativa y cultural, entre ellos los servicios de salud. En la Ley 100 de 1993 el Estado fijó su responsabilidad de intervenir en el actual mercado de la salud, en aras de garantizar 
la participación social como fundamento de la prestación del servicio público de la salud. Así, la participación ciudadana en Colombia es considerada como la posibilidad que los ciudadanos, comunidades y grupos sociales tienen de incidir en las políticas públicas, sin pasar necesariamente por la democracia representativa, los partidos políticos, las prácticas clientelistas o la organización gremial (4).

En el pasado reciente, en Colombia, investigadores se han interesado por indagar sobre el derecho a la salud y el acceso efectivo a los servicios de salud (5-9). Sin embargo, pocos han valorado la percepción de los ciudadanos sobre el derecho a la salud a partir de conocer el acceso real a los servicios. En la presente investigación se pretende conocer la percepción que tienen los ciudadanos usuarios de RS del acceso a los servicios de salud y a la participación social como elementos esenciales para el logro de su derecho a la protección sanitaria.

\section{MATERIALES Y MÉTODOS}

Se llevó a cabo un estudio transversal, con el propósito de conocer la percepción de los usuarios del RS en el municipio de Arjona, Bolívar, Colombia, respecto al acceso a los servicios y el derecho a la salud, en el marco del proyecto: "Asistencia Técnica Para el Fortalecimiento Institucional de las Entidades Territoriales en la Gestión del Régimen Subsidiado, Zona Costa Atlántica” del Programa de Apoyo a la Reforma en Salud del Ministerio de la Protección Social de Colombia.

Población y muestra

El universo estuvo integrado por los $27197^{\mathrm{a}}$ afiliados al RS en el municipio de Arjona, distribuidos entre 6 EPS del RS. Se definió una muestra de 600 beneficiarios fijando un nivel de confianza del $95 \%$ y un error máximo esperado de $5 \%$. La Tabla 1 muestra la fracción de muestreo y el número de afiliados a encuestar para cada uno de las EPS del Régimen Subsidiado del municipio de Arjona.

\begin{tabular}{|c|c|c|c|}
\hline $\begin{array}{c}\text { EPS del Regimen } \\
\text { Subsidiedo }\end{array}$ & $\begin{array}{c}\text { Total } \\
\text { Afiliado8 }\end{array}$ & $\begin{array}{c}\text { Fracción } \\
\text { de muestreo }(\%)\end{array}$ & $\begin{array}{l}\text { Afiliados a } \\
\text { encuestar }\end{array}$ \\
\hline COMFAMILIAR & 4488 & 16.5 & 99 \\
\hline SOLSALUD & 3672 & 13.5 & 81 \\
\hline MUTUAL SER & 8023 & 29.5 & 177 \\
\hline COMPARTA & 3318 & 12.2 & 73 \\
\hline COOSALUD & 3345 & 12.3 & 74 \\
\hline EMDISALUD & 4352 & 16.0 & 96 \\
\hline TOTAL & 27197 & 100.0 & 600 \\
\hline
\end{tabular}

a Datos suministrados por la Secretaría de Salud Municipal de Arjona, Bolívar.2005 
Recolección y análisis de datos

Los datos fueron recolectados utilizando un formulario estandarizado y validado previamente respecto a su validez de constructo. Este fue aplicado por los funcionarios de la Secretaría de Salud previamente capacitados, a los afiliados al RS en las salas de espera de las oficinas de atención al usuario de las distintas EPS del RS, durante el segundo semestre del año 2005. Dicho formulario integró información sobre el conocimiento que tienen los afiliados acerca del proceso de identificación, selección y priorización de beneficiarios al RS, percepciones acerca de: a) aseguramiento del riesgo, b) calidad de la atención, c) acceso a los servicios y d) participación comunitaria. Las opciones de respuestas tuvieron una secuencia ordinal (p.e: "nunca”, "algunas veces” "muchas veces”). Las encuestas fueron tabuladas en el paquete estadístico SPSS 11.0®, versión en español. Para valorar la relación entre la percepción acerca de la calidad de la atención de la EPS del RS y la participación de los afiliados en las actividades de las mismas, se utilizó la medida de asociación no paramétrica Tau-b de Kendall (10).

\section{RESULTADOS}

Percepción de la participación en los procesos de identificación, selección y priorización de los potenciales beneficiarios del RS

El desconocimiento y la falta de información se hacen evidentes en las respuestas. Dos tercios de los encuestados manifestaron no estar en la capacidad de afirmar con que frecuencia se publican las listas municipales de priorizados mientras que el $15 \%$ afirma que nunca se publica dichas listas. Resultados similares se encontraron al interrogar a los usuarios acerca de la verificación de las listas censales. Entre los pocos usuarios que opinan en torno a la participación ciudadana en la validación de las listas publicadas más de la mitad de ellos tiende a creer que fueron ignorados en el levantamiento de las listas del SISBEN. Así mismo, el conocimiento de la existencia de los listados censales en el municipio es casi nulo debido a que al parecer la Dirección Local de Salud no los divulga. En el mismo sentido, al parecer las Ligas de Usuarios no han difundido y no han sensibilizado a los usuarios respecto al uso de mecanismos de verificación de listados censales (el $94 \%$ de los encuestados dice no conocer ningún mecanismo de verificación). 
Percepción del aseguramiento brindado por las EPS del RS

Existe un convencimiento acerca de las bondades de estar afiliado al RS. El 63 $\%$ de los encuestados considera que el RS le garantiza el derecho a la salud. De la misma manera, el 67 \% de los encuestados manifestó conocer las formas de acceso al RS.

El $47 \%$ de los usuarios encuestados considera que la inclusión en el RS es producto de un derecho, el $20 \%$ de los encuestados considera que es por gestión de la alcaldía, mientras que el $11 \%$ considera que la inclusión de las personas es gracias a la gestión comunitaria y el 15 \% de la población encuestada atribuye a terceros (amigos y políticos) el acceso al aseguramiento en el RS.

En cuanto a la efectividad del aseguramiento, dos tercios de los encuestados manifestaron desconocer la red prestadora de servicios de salud de la EPS del RS a la cual están afiliados. En cada EPS del RS menos de la mitad de los afiliados encuestados conoce la red prestadora de servicios de salud.

El $15 \%$ de los encuestados considera que algunas veces las EPS del RS han defendido sus derechos y otro $15 \%$ considera que la defensa de sus derechos ha sido mas frecuente, el resto de los usuarios encuestados no se sienten capacitados para opinar respecto al papel que juega la EPS del RS en cuanto a la defensa de sus derechos.

Percepción de los usuarios acerca del acceso a los servicios de salud

El $53 \%$ de los encuestados considera difícil acceder a los servicios de salud. El $16 \%$ de los afiliados encuestados afirma que es muy fácil acceder a los servicios de salud y el $1 \%$ considera imposible acceder a estos mismos servicios. En cuanto a identificación de barreras de acceso a los servicios de salud, el 36 \% de los encuestados considera a las barreras de tipo administrativo (los trámites ante las EPS del RS) como el obstáculo más importante para acceder al servicio; Uno de cada 3 encuestados afirma que las barreras geográficas (distancia de la vivienda) es la principal de las barreras y el $20 \%$ considera que las barreras financieras (copagos) dificultan el acceso a los servicios.

Percepción de los usuarios acerca de la calidad en la prestación de los servicios de salud 
A la hora de evaluar cada una de las EPS del RS, los usuarios expresan que los ejercicios de evaluación de satisfacción de usuarios por parte de las EPS del RS en el municipio son esporádicos. Ninguna de las EPS del RS es vista como receptora de las inquietudes de los usuarios.

Al indagar acerca de la opinión en cuanto a la oportunidad del servicio, uno de cada tres usuarios encuestados contestó que algunas veces es atendido oportunamente, el $21 \%$ respondió que siempre es atendido a tiempo y el $10 \%$ calificó la atención de inoportuna. Un tercio de los encuestados no opinó respecto al tema.

Por otro lado, más de la mitad de los afiliados en todas la EPS del RS del municipio no conocen los programas de promoción y prevención.

Percepción de los usuarios acerca de la participación comunitaria

Los resultados son consistentes con la escasa participación en los procesos de identificación, selección y priorización de beneficiarios. La quinta parte de los encuestados no conoce ningún caso de múltiple afiliación, así como una proporción similar dice que estos casos se presentan con muy poca frecuencia, y cerca del $8 \%$ considera que si acontecen con mucha frecuencia. Respecto a la percepción de la participación de los afiliados en las actividades de las Instituciones Prestadoras de Servicios de Salud-IPS- cerca del $40 \%$ de los encuestados la califica entre mala y poco significativa mientras que el $20 \%$ la considera como decisiva en pro de los usuarios.

La percepción que tienen los usuarios acerca de la calidad de la atención de las EPS del RS se encuentra correlacionada positivamente con el nivel de participación de los afiliados en las actividades de dichas empresas. La medida no paramétrica de Tau-b de Kendall arroja un valor de 0,330 ( $\mathrm{p}=0,00)$. Es decir, la creencia acerca de la calidad de atención de las EPS del RS es explicada aproximadamente en una tercera parte debido a la inclusión activa de los usuarios en las actividades de estas empresas.

\section{DISCUSIÓN}

En Colombia se reconoce la salud como un "derecho negativo", es decir, las personas tienen derecho a exigir que su vida e integridad física sean respetadas pero no significa que exista un derecho al cuidado de la salud(5). En este sentido, se tiende a usar el termino "derecho a la salud" en aquellos documentos que 
hacen alusión a los de derechos humanos, lo cual puede interpretarse como derecho a "ser saludable", es decir, no poseer enfermedades, en lugar de expresar el derecho a acceder a los bienes y servicios necesarios para alcanzar el más alto nivel posible de salud (11).

De otro lado, la asistencia del Estado en función de la falta de capacidades (gratuita para pobres y no gratuita para quienes tienen capacidad de pago), impone valorar la pobreza económica de los posibles beneficiarios (12). La participación comunitaria es importante en el proceso de redistribución de recursos para grupos vulnerables de la población y en consecuencia garantizar el derecho a la salud (13). Inclusive, hoy día existe una tendencia mundial de utilizar la participación comunitaria como un condicionante de la financiación de diversos proyectos (14) al tiempo que debe estar encaminada a que las poblaciones regulen los procesos de identificación de beneficiarios, lo que se conoce como autofocalización. Se dice que la autofocalización tiene entre sus ventajas que está menos sujeta a la corrupción y manipulación burocrática, es más aceptable políticamente porque son las mismas personas las que toman las decisiones de participación y se adapta más fácilmente a las cambiantes circunstancias de los pobres. Además, permite obtener mejores resultados a un costo muy bajo. Pero esta autofocalización debe basarse en el comportamiento colectivo y no en el individual como suele suceder (15).

En Arjona, Bolívar, se observa un desconocimiento acerca de la participación en los procesos de identificación, selección y priorización de los potenciales beneficiarios del RS. La comunidad ha asumido solamente el papel de beneficiaria y desconoce el crucial rol que está llamada a ejercer como intermediaria e interventora de los distintos procesos que hacen parte de la gestión local del RS. En la región predomina la falta de sustento técnico en los municipios a la hora de elaborar las listas de la población beneficiaria del régimen subsidiado y es fácilmente encontrar evidencias acerca de la manipulación del SISBEN para ingresar personas no merecedoras del aseguramiento subsidiado (16).

La actitud que tiene la Secretaría de salud de no involucrar a la ciudadanía en los procesos de identificación, selección y priorización profundiza la tendencia natural del ciudadano a no considerar que el uso del subsidio por parte de quienes no deben, redunda en el detrimento de los derechos de los que si necesitan el subsidio. Lo anterior se refleja en que existe un demarcado interés en cuanto a la afiliación individual pero no hay una conciencia colectiva acerca de los procesos de identificación, selección y priorización. Esto es consistente con el señalamiento 
de algunos autores acerca de la concepción que se tiene en cuanto al derecho a la salud como un problema de acceso individual a servicios de atención médica (7).

La percepción del seguro del RS es similar a lo hallado en una investigación acerca de accesibilidad en este mismo grupo poblacional realizada por el grupo de Economía de la Salud-GES de la Universidad de Antioquia en la cual de igual manera se reconoce el alcance del aseguramiento (17).

Todas las EPS del RS del municipio están niveladas por lo bajo en cuanto al conocimiento de la red prestadora de servicios por parte de sus afiliados. No hay la suficiente información y difusión a los usuarios de los sitios en donde pueden ser atendidos. Es decir, el papel de agencia que cumplen las EPS del RS evaluado desde el punto vista de los usuarios es desconocido y por ende no es siquiera percibido. Del mismo modo, existe incapacidad por parte de los usuarios a la hora de calificar la oportunidad en la atención, dando entender que no están acostumbrados a participar en ejercicios semejantes en donde se valoren sus percepciones. Al respecto, es pertinente preguntarse si las EPS del RS cumplen su rol de asegurador y no solo con la administración de subsidios.

Los afiliados entienden la participación en el sistema de salud como el ejercicio de adelantar acciones con el propósito de recibir un servicio. Así el éxito de sus gestiones se encuentra asociada con la "bondad" del funcionario de turno de la secretaría de salud municipal y esto distorsiona la verdadera concepción del derecho a la salud, desde el principio de ciudadanía.

Con la información obtenida por estudios como el presente es fácil construir un ranking de las EPS del RS basado en la oportunidad de la atención según sus propios usuarios, así como un ranking basado en la percepción de calidad de atención. Estos indicadores deben ser socializados con la comunidad a fin de que se tenga mayor información a la hora de decidir la institución que le otorgará el aseguramiento en salud.

El país carece de indicadores que midan el estado actual del derecho a la salud. En su defecto se usan cifras relacionadas con los recursos destinados a salud y su gestión. Así, los logros en materia de cobertura del aseguramiento en salud deben significar, no solo población asegurada, sino acceso efectivo a servicios de salud suficientes y de calidad. En tal sentido, la opinión de la comunidad, en especial la de los sectores sociales más vulnerables, puede constituirse en indicador del cumplimiento del derecho a la salud * 
394 REVISTA DE SALUD PÚBLICA · Volumen 10 (3), Julio 2008

\section{REFERENCIAS}

1. Organización Panamericana de la Salud, Derechos Humanos y Derecho a la Salud: Construyendo Ciudadanía en Salud. Marco conceptual, aspectos metodológicos y alcances operativos. En: Ministerio de Salud de Perú, Lima. Cuaderno de promoción de la salud; 2005p. 102.

2. Gosepath S. Consideraciones sobre las fundamentaciones de los derechos humanos sociales. En: Alonso MA, Ramírez JG (Eds.). Ciudadanía y derechos humanos sociales. Medellìn; Escuela Nacional Sindical; 2001. p. 15-58.

3. Organización Mundial de la Salud, Oficina del Alto Comisionado para los Derechos Humanos, Pacto Internacional de Derechos Económicos, Sociales y Culturales. Ginebra; 1966.

4. Restrepo DI. Participación Social: Relaciones Estado Sociedad Civil. Rev. salud pública (Bogotá). 2001;3:245-267.

5. Álvarez LS. The right to health in Colombia: a proposal for laying down its moral foundations. Rev Panam Salud Pública. 2005; 18(2):129-35.

6. Paredes NN. El derecho a la salud: Su situación en Colombia. Cinep-GTZ. 2003; p. 115.

7. Hernández M. Health reform, equity and the right to health in Colombia. Cad Saude Publica. 2002; 18(4): 991-1001.

8. Arbeláez M. Evaluación de la eficacia del derecho a la salud en Colombia a partir del proceso de descentralización sanitaria. Derecho y salud. 2007; 15(1): 29-74.

9. Arbeláez M. La protección constitucional del derecho a la salud: la jurisprudencia de la Corte Constitucional Colombiana.Derecho y salud. 2006; 14(2):205-240.

10. Liebetrau AM. Measures of Association Series: Quantitative Applications in the Social Sciences.: Sage Publications Inc. 95; 1983.

11. Lucía M. Derecho a la salud en México. Un análisis desde el debate teórico contemporáneo de la justicia sanitaria. Revista IIDH. 2004; 40: 291-313.

12. Sen A. Libertad y Desarrollo. Bogotá: Editorial Planeta. 2000; p.168-170.

13. Conning JKM. Mecanismos de selección para las redes de seguridad social basadas en la comunicación. Instructivo del Banco Mundial sobre redes de Seguridad. Banco Mundial; 2001.

14. Ravallion M, Wodon Q. Evaluating a Targeted Social Program when Placement is Decentralized. The World Bank. Development Research Group. Policy Research Working Paper; 1998. p. 15.

15. Behrman J. Focalización de la Protección Social a los pobres ¿Cuáles son los "Tradeoffs"? Banco Interamericano de Desarrollo. Washington, D.C.; 2001. p. 31-34.

16. Ruiz F, Acosta N, Ardila Z, Eslava J, Peñaloza E, Puente C, Reyes S. Entorno, aseguramiento y acceso en el Régimen Subsidiado en Colombia. Seis estudios de caso. Santafé de Bogotá: Fundación Corona y Cendex; 1999. p. 166.

17. Restrepo JH. Condiciones de acceso y sostenibilidad financiera del régimen subsidiado de salud en Antioquia. Medellín: Universidad de Antioquia Centro de Investigaciones Económicas-CIE: 2003. 